\title{
PML induces a novel caspase-independent cell death process
}

Fredérique Quignon et al.

Nature Genet. 20, 259-265 (1998).

A fraction of Bax was suggested to be associated with PML nuclear bodies based on colocalizations of Bax and PML using several antibodies. The Bax antibody (SC I-19) yielding the strongest nuclear-body signal has since been shown to cross-hybridize with a conformation-specific epitope of the nuclear body-associated Sp100 protein. Given the weaker nuclear-body signal observed with the other antibodies and awaiting further evidence, we feel that at this stage Bax cannot be considered as a nuclear-body antigen. This particular point does not change any of the other evidence or conclusions presented in the article. 